\title{
Comparação entre os dados de vento das reanálises meteorológicas ERA-Interim e CFSR com os dados das estações automáticas do INMET no Rio Grande do Sul
}

\section{Comparison of wind data of ERA-Interim reanalysis and CFSR with the data from automatic INMET stations in Rio Grande do Sul}

\author{
Eduardo Stüker ${ }^{1}$, Cristiano Henrique Schuster ${ }^{1}$, Jean Jonathan Schuster ${ }^{1}$, Daniel Caetano \\ Santos$^{2}$, Luis Eduardo Medeiros ${ }^{1}$, Felipe Denardin Costa', Giuliano Demarco ${ }^{2}$ e Franciano \\ Scremin Puhales ${ }^{2}$ \\ ${ }^{1}$ Universidade Federal do Pampa \\ eduardostuker@gmail.com; cris.h.schuster@gmail.com; jeanjschuster@gmail.com;luizmedeiros@unipampa.edu.br; fdenardin@ \\ unipampa.edu.br; \\ ${ }^{2}$ Universidade Federal de Santa Maria \\ danielcae@gmail.com; giulianofisico@gmail.com; franciano.puhales@ufsm.br
}

\begin{abstract}
Resumo
A energia eólica é, atualmente, uma das fontes de eletricidade que mais crescem em todo o mundo. Porém, especialmente no Brasil, ainda é muito difícil a localização de regiões com ventos confiáveis para a implantação de um parque eólico pois não existe uma densidade de dados válidos que sirvam de garantia de eficiência do parque. Assim, o desenvolvimento de modelos que simulam as condições de vento são extremamente importantes para estudos e pesquisas na área. Neste sentido, dados de reanálises meteorológicas podem servir de entrada para o desenvolvimento de modelos regionais de alta resolução, por exemplo. Portanto, este trabalho apresenta um estudo de comparação de dois conjuntos de dados de vento de Reanálises meteorológicas - ERA-Interim e CFSR - com dados medidos nas estações meteorológicas automáticas do Instituto Nacional de Meteorologia no estado do Rio Grande do Sul com o propósito de obter o coeficiente de correlação dos dados das reanálises com os dados medidos para cada ponto de medição e para cada estação do ano. Para melhor visualização dos resultados de correlação, também é construido mapas de contorno com coeficiente de correlação onde observa-se um melhor desempenho da reanálise do CFSR.
\end{abstract}

Palavras-chave: Energia Eólica, Campo de Vento, Reanálises Meteorológicas, ERA-Interim, CFSR..

\begin{abstract}
Wind power is currently one of the sources of electricity the fastest growing worldwide. However, especially in Brazil, it is still very difficult to locate areas with reliable winds for the implementation of a wind farm, because there is not enough data density that guarante the wind farm efficiency. Thus, the development of models that simulate wind conditions are extremely important for studies and research in this area. In this way, weather reanalysis data can be used as input into high-resolution regional models, for example. Therefore, this work presents a study comparing two sets of weather Reanalysis of wind data - ERA-Interim and CFSR - with measured data from automated weather stations of the National Institute of Meteorology in the Rio Grande do Sul state in order to obtain the coefficient correlation of data from reanalysis with data measured for each measurement point and for every season. For better visualization of the correlation results, it is also built contour maps with correlation coefficient where can be seeing that the best performance of the CFSR reanalysis.
\end{abstract}

Keywords: Wind Energy, Wind Field, Reanalysis, ERA-Interim, CFSR. 


\section{Introdução}

A energia eólica é hoje uma das principais fontes de eletricidade estudadas e pesquisadas em todo o mundo. No Brasil está em ampla expansão, segundo a Câmara de Comercialização de Energia Elétrica (CCEE) as usinas eólicas brasileiras aumentaram em $114 \%$ a produção de energia no primeiro semestre de 2015. A geração média nesse período foi de 1.840 MW médios frente aos 860 MW médios alcançados no mesmo período de 2014. No fim de junho de 2014 a fonte era responsável por 1,4\% do total gerado no Brasil, atualmente representa 3\% (CCEE, 2015).

A capacidade instalada das 244 usinas eólicas cadastradas na CCEE chegou a 6.211 MW ao final do primeiro semestre de 2015. O crescimento é de $60 \%$, em relação ao mesmo período do ano passado, quando a capacidade das 156 instalações em operação era de 3.891 MW. Os dados consolidados da CCEE apontam que o Rio Grande do Norte com 2.104 MW segue na liderança em capacidade instalada da fonte, seguido por Ceará (1.301 MW), Rio Grande do Sul (1.300 MW) e Bahia (959 MW) (CCEE, 2015). Isso demonstra que a fonte eólica esta cada vez mais presente no mercado brasileiro de energia, mostrando ser uma fonte competitiva e rentável para seus investidores. Mas, mesmo com esse crescimento exponencial, ainda existe muito potencial não explorado nem estudado como deveria. A identificação de locais favoráveis para a implantação de um parque eólico ainda é muito difícil, principalmente em regiões em que não existe uma grande densidade de dados de vento.

A estimativa do potencial eólico bruto é comumente obtida por meio de medições locais de vento, que devem ser registradas por no mínimo três anos. No entanto, um período de medições expandido em até cinco anos ainda é curto, se for comparado a períodos normais para a definição de uma média climatológica de no mínimo 30 anos. Assim, são grandes as incertezas quanto ao verdadeiro potencial eólico da região. Além disso, o planejamento, a instalação e a manutenção de antenas de medição de vento é um empreendimento caro, na hipótese da região não se mostrar um promissor local para geração eólica, uma quantidade considerável de investimento é irreversivelmente perdida (Carvalho et al., 2013; Pinto, 2013). A utilização destas medições ainda é um obstáculo importante em países em desenvolvimento, que não têm uma rede densa de estações meteorológicas. Como resultado, a forma mais comum para caracterizar o vento é a interpolação dos dados para um ponto específico. No entanto, este método tem validade limitado, porque, em muitos casos, a interpolação só pode ser realizado quando as características de relevo são uniformes.

Uma possível solução para a identificação de locais com potencial para a instalação de um parque eólico é a utilização de simulações numéricas realizadas por modelos atmosféricos, tais como modelos meteorológicos de mesoescala. Esses modelos permitem simulação do campo de vento regional, além de outras variáveis meteorológicas importantes, baseando-se na integração de informações de medições meteorológicas, e informações complementares, tais como topografia, rugosidade e perfis de vento verticais, entre outros (Stensrud, 2007).

Um conjunto de dados de entrada muito importante para um modelo regional de simulação de campo de vento podem ser os dados provenientes de reanálises meteorológicas, que são conjuntos de dados em grade, que combinam os dados medidos e fornecidos pelos institutos nacionais de meteorologia com dados obtidos a partir de modelos de circulação global de alta resolução no estado da arte, fornecendo uma síntese das observações disponíveis em todo o mundo no contexto de um modelo físico (Kalnay et al., 1996). Dessa forma, o objetivo desse trabalho é analisar a validade de duas reanálises meteorológicas (descritas nas seções 2.1.1 e 2.1.2), amplamente utilizadas na literatura, para o estado do Rio Grande do Sul, comparando-as com dados das estações meteorológicas automáticas do Instituto Nacional de Meteorologia (INMET).

\section{Materias e Métodos}

\subsection{Reanálises}

Reanálise meteorológica, como defindo anteriormente, é um conjunto de dados obtidos a partir de modelos de circulação global com dados medidos, sintetizando os dados disponíveis em um contexto físico. Atualmente, existem duas gerações de dados de reanálises. A primeira compreende três principais conjuntos de dados: $\mathrm{O}$ NCEP-R1 produzido e lançado pelo National Oceanic and Atmospheric Administration (NCEP); O ERA-40 do European Centre for Medium-Range Weather Forecasts (ECMWF) e; JRA-25 da Japanese Meteorological Agency.

A fim de corrigir vários problemas identificados no NCEP-R1, uma segunda versão foi lançada pelo NCEP, o NCEP-R2. Que é a única reanálise de primeira geração processada operacionalmente até hoje. A ERA-40 foi encerrada em 2001 e a JRA-25 em 2004. Recentemente uma nova geração de reanálises foram produzidas e lançadas (Carvalho et al., 2013): ERA-Interim do ECMWF (Dee et al., 2011); CFSR do NCEP (Saha et al., 2010); MERRA da NASA (Rienecker et al., 2011). Essa nova geração de reanálises proporcionou um progresso significativo, devido aos avanços operacionais na previsão do tempo e também nos esforços feitos para melhorias com base nas reanálises anteriores.

Tendo em vista que o objetivo deste trabalho é a análise da validade dos dados de reanálise, no estado 
do Rio Grande do Sul, são utilizadas as reanálises ERAInterim e CFSR que fazem parte da nova geração de reanálises. Essas reanálises foram escolhidas, por dois motivos: ambas estão disponíveis e têm facilidade de acesso e, além disso, alcançaram bons resultados em estudos, similares ao aqui proposto, que comparam as diferentes reanálises com dados de medições locais de vento, (Liléo e Petrik, 2011; Carvalho et al., 2013).

\subsubsection{ERA-Interim}

O ERA-Interim é a reanálise atmosférica global mais recente produzida pelo ECMWF, e foi lançada em 2009. Possui uma série de melhorias quando comparado com seus antecessores ERA-15 e ERA-40. Abrange o período a partir de 1 de janeiro de 1979, e continua a ser desenvolvido até hoje. Foi originalmente concebido para melhorar o período entre os anos de 1990 e 2000 onde passou a existir uma quantidade maior de dados disponíveis. A resolução horizontal do modelo atmosférico é de aproximadamente $79 \mathrm{~km}$ em uma grade gaussiana reduzida. É atualizado duas vezes por dia (0000 UTC e 1200 UTC), mas oferece dados de seis em seis horas (0000 UTC, 0600 UTC, 1200 UTC e 1800 UTC). (Dee et al., 2011)

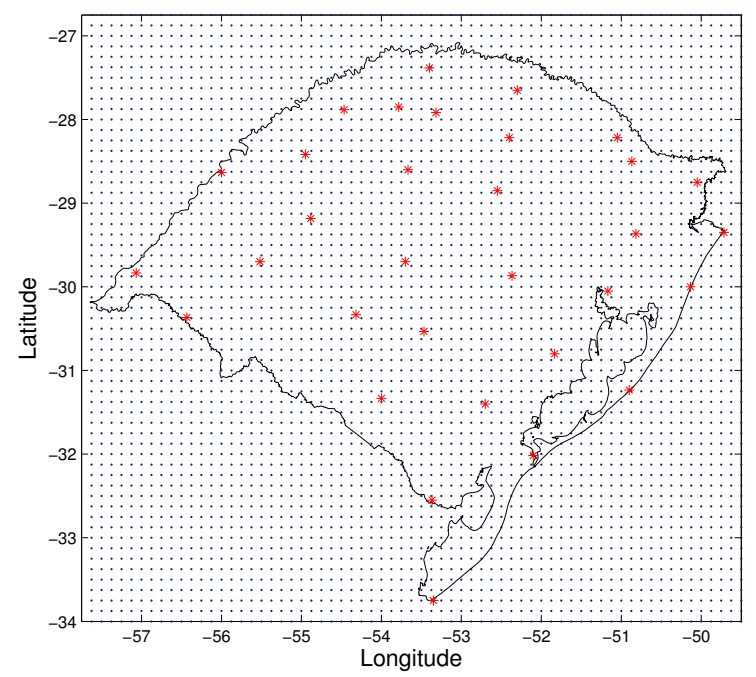

Figura 1: Mapa com os pontos de grade da reanálise ERA-Interim com as 33 estações automaticas do INMET analisadas.

Para o desenvolvimento deste trabalho foram utilizadas as séries temporais (1979-2014) das componentes zonal (leste-oeste, denotada por $u$ ) e meridional (nortesul, denotada por $v$ ) do vento a dez metros de altura extraídos do ponto de grade mais próximo da estação do INMET. Essas séries são amostrados a cada seis horas e, tem uma grade de 0,125 x 0,125 graus. A Figura 1 apresenta o mapa do Rio Grande do Sul com os pontos de grade do ERA-Interim e as estações automáticas do INMET.

Como o objetivo é fazer a comparação da magnitude $(\bar{M})$ e direção $(\phi)$ do vento, e o ERA-Interim fornece apenas as componentes $u$ e $v$, são utilizadas as equações 1 e 2 para obter $\bar{M}$ e $\phi$ respectivamente.

$$
\bar{M}=\sqrt{u^{2}+v^{2}}
$$

$$
\phi=-\operatorname{actg}\left(\frac{u}{v}\right)
$$

\subsubsection{CFSR}

Completada em agosto de 2010, a reanálise do CFSR compreende um período de 31 anos entre 1979 e 2010. Possui uma resolução vertical e horizontal da atmosfera superior comparado a reanálises anteriores. São aproximadamente $38 \mathrm{~km}$ na horizontal com 64 níveis na vertical e resolução temporal de seis horas. Um grande avanço dessa reanálise meteorológica foi o monitoramento em tempo real que ocorreu durante a sua execução. Milhares de análises foram geradas automaticamente no final de cada mês reanalisado. Várias fontes de dados foram utilizadas para o seu desenvolvimento. Muitos arquivos históricos, operacionais e conjuntos de observações recém processadas que estão sendo produzidos em centro de pesquisas em todo o mundo. As observações históricas são muitas vezes encontradas

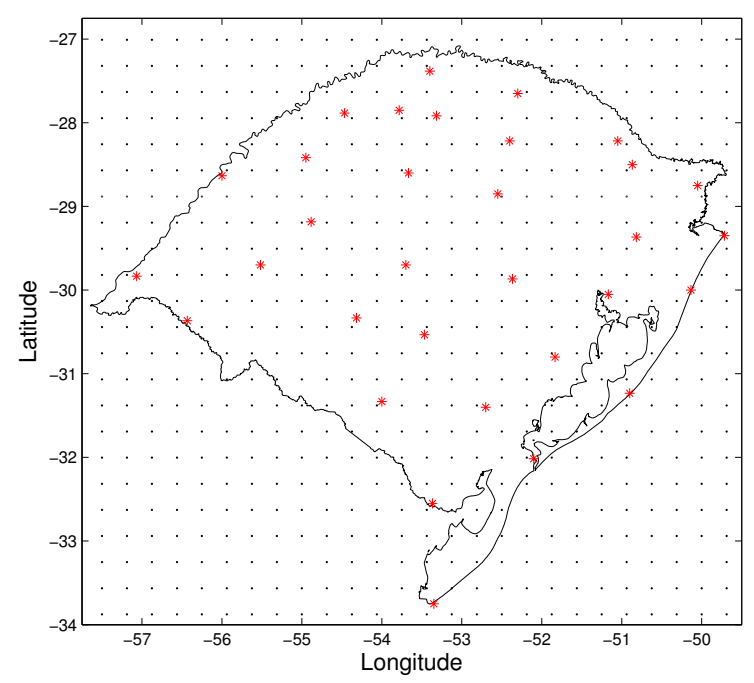

Figura 2: Mapa com os pontos de grade da reanálise CFSR com as 33 estações automaticas do INMET analisadas. 
armazenadas em formatos obsoletos além de serem encontradas em vários estágios de desordem e incompletas. Uma grande garimpagem foi realizada em diversos institutos de meteorologia, pesquisa e militar. Além disso, uma série de conjuntos de dados modernos e importantes foram reprocessados, o que melhorou o resultado final da reanálise. (Saha et al., 2010)

Assim como para a reanálise ERA-Interim (seção 2.1.1), para a CFSR foram utilizadas as séries temporais de $u$ e $v$ a dez metros e calculado a magnitude e direção do vento através as equações 1 e 2 respectivamente. O período de tempo utilizado foi de 1979 até 2009 e, como mostra a Figura 2, com grade de 0,5 x 0,5 graus. Esses dados também são amostrados a cada seis horas, coincidindo com os horários da reanálise do ECMWF.

\subsection{Estações automáticas do INMET}

As estações meteorológicas automaticas do INMET (EMA) coletam, de minuto em minuto, as informações meteorológicas (temperatura, umidade, pressão atmosférica, precipitação, direção e velocidade de vento e radiação) para a área onde estão localizadas. No estado do Rio grande do Sul são 37 EMA's distribuídas por todo o seu território. Nas Figuras 1 e 2 pode ser visto a distribuição das 33 EMA's utilizadas nesse estudo. É importante destacar, que as estações de Campo Bom, Dom Pedrito, Ibirubá e Teutônia não foram consideradas por não haver dados suficientes. Todas as estações são instaladas em bases fixas, numa área livre de obstruções naturais e prediais, situadas em uma área mínima de $14 \mathrm{~m}$ por 18 $\mathrm{m}$. Os sensores e demais instrumentos são fixados em um mastro metálico de dez metros de altura.

As EMA's tem amostragem de vento a cada segundo, sendo que os relatórios meteorológicos utilizam valores médios de dez minutos. O transmissor de vento utilizado realiza medidas a cada 0,25 segundos para calcular rajadas e a média móvel de três segundos tanto para a magnitude quanto para direção do vento. A EMA recebe esses valores e os usa como variáveis de entrada para calcular as médias de 10 minutos e as velocidades de rajada (INMET, 2011).

O INMET disponibiliza, além dos dados em tempo real das estações convencionais e automáticas, um banco de dados denominado Banco de Dados Meteorológicos para Ensino e Pesquisa - BDMEP, destinado a apoiar as atividades de ensino e pesquisa. Esse banco de dados abriga dados meteorológicos diários em formato digital, referente às medições diárias, de acordo com as normas técnicas internacionais da Organização Meteorológica Mundial. Os dados de vento $(\bar{M}$ e $\phi)$ são amostrados de hora em hora. Dessa forma, para este trabalho, são utilizados apenas os horários que coincidem com os dados das reanálises, ou seja, 0000 UTC, 0600 UTC, 1200 UTC e 1800 UTC.

\subsection{Análise de Correlação}

O coeficiente de correlação $(R)$ indica o grau de ajuste dos dados das reanálises aos dados observados, sendo que $R=1$, ou $100 \%$, corresponde a um concordância perfeita entre o simulado e o observado e $R=0$, ou $0 \%$, corresponde a uma discordância total entre os dados e pode ser calculado através da equação (3) (Hanna, 1988).

$$
R=1-\frac{\frac{1}{n} \sum_{i=1}^{n}\left(\varepsilon_{s}-\varepsilon_{o}\right)^{2}}{\frac{1}{n} \sum_{i=1}^{n}\left(\left|\varepsilon_{s}-\bar{\varepsilon}\right|+\left|\varepsilon_{o}-\bar{\varepsilon}\right|\right)^{2}}
$$

onde $n$ é o número de observações, $\varepsilon_{s}$ é o dado da reanálise, $\varepsilon_{o}$ é o dado observado (INMET) e $\bar{\varepsilon}$ é a média dos dados observados.

É importante destacar que as lacunas existentes nos dados das EMA's foram desconsiderados. Além disso, os períodos de comparações foram os mesmos disponíves por cada EMA para cada conjunto de reanálises utilizadas na comparação.

\section{Resultados e Discussões}

Inicialmente obteve-se os coeficiente de correlação, calculado através da equação 3, de cada reanálise em relação aos dados de cada uma das EMA's. Este procedimento foi aplicado tanto para magnitude quanto para direção do vento. $\mathrm{O}$ coeficiente de correlação médio da magnitude do vento, para todo o período analisado, para a reanálise do CFSR foi de $R=0,7127$, enquanto que para a reanálise do ERA-Interim o coeficiente médio de correlação foi de $R=0,6965$. Já os resultados da correlação entre as reanálises e as EMA's para direção do vento, se mostraram inferiores e com um melhor desempenho do ERA-Interim com $R=0,3642$, equanto o CFSR obteve um coeficiente $R=0,3331$.

A EMA com melhor resultado para a magnitude do vento foi a mesma, tanto para o ERA-Interim quanto para o CFSR, e está localizada no município de Jaguarão no sudeste do RS com correlações de $80,26 \%$ e $81,42 \%$ respectivamente. Os coeficientes com piores resultados foram nas EMA's de Cruz Alta no Noroeste e de São Borja no sudoeste do RS com correlações de 57,22\% e de $53,63 \%$ para o ERA-Interim e para o CFSR respectivamente.

Já para a direção do vento os melhores resultados foram nas EMA's de Tramandaí no litoral norte do RS com $R=0,4921$ e de Mostardas também no litoral norte do estado com $R=0,4847$ para o ERA-Interim e para o CFSR respectivamente. Os piores resultados foram, respectivamente, de apenas $19,68 \%$ e $10,08 \%$ para o ERAInterim em Santo Augusto no noroeste e para o CFSR em São José dos Ausentes no nordeste do estado.

No que concerne a análise das estações do ano, como pode ser visto nas Tabelas 1 e 2, os resultados foram 
semelhantes para as duas reanálises, tanto para a magnitude (Tabela 1) quanto para a direção (Tabela 2) do vento.

Tabela 1: Coeficiente de correlação da magnitude do vento

\begin{tabular}{lcccc}
\hline Reanálise & Verão & Outono & Inverno & Primavera \\
\hline ERA & 0,6856 & 06993 & 0,7016 & 0,6996 \\
CFSR & 0,7089 & 0,7125 & 0,7218 & 0,7074 \\
\hline
\end{tabular}

Tabela 2: Coeficiente de correlação da direção do vento

\begin{tabular}{lcccc}
\hline Reanálise & Verão & Outono & Inverno & Primavera \\
\hline ERA & 0,3359 & 0,3850 & 0,3971 & 0,3387 \\
CFSR & 0,3283 & 0,3455 & 0,3826 & 0,2758 \\
\hline
\end{tabular}

Observa-se que os piores coeficientes de correlação estão nos períodos da primavera e verão, e que no inverno encontram-se os melhores resultados gerais, tanto para a magnitude como para a direção do vento. Também verifica-se, que o CFSR tem melhor desempenho para a magnitude do vento enquanto que o ERA-Interim tem melhor resultado para a direção do vento.

Os resultados médios obtidos para cada EMA são melhor visualizados através do mapa mostrado nas Figuras 3 e 4 . A parte colorida dos mapas representam os coeficientes de correlação conforme a barra de cores vertical no lado direito do mapa, a parte cinza escuro é desconsiderada por não existir estações para além da fronteira entre a área colorida e a área cinza escuro, não podendo assim ser calculada a interpolação da correlação para esses pontos.

As Figuras 3(a) e 3(b) apresentam os mapas de coeficientes de correlação para a magnitude do vento no período do inverno para o ERA-Interim e CFSR respectivamente. Dessa forma, pode-se comparar o desempenho das duas reanálises para o mesmo período.

Por conseguinte, observa-se que a reanálise do CFSR obteve desempenho melhor comparada com a do ERAInterim. Os melhores resultados da magnitude do vento para o CFSR estão localizados principalmente nas regiões Sul, entre as regiões da Fronteira Oeste e Centrooeste, Fronteira Sudoeste e Centro-Nordeste com correlações de $80 \%$ a $85 \%$. O ERA-Interim apresenta correlaç̃̃es parecidas em apenas dois pontos, na região Sul e entre as regiões da Fronteira Oeste e Centro-oeste.

Nas Figuras 4(a) e 4(b) é mostrado os mapas de correlação para a direção do vento. Pode-se observar que a correlação é fraca em todo o estado para as duas reanálises, não passando de 55\%. CFSR e ERA-Interim apresentam resultados muito semelhantes, tendo as maiores correlações no Litoral e Fronteira Oeste. De modo geral, o ERA-Interim demonstra uma leve vantagem, pois em três ponto do mapa do CFSR (Figura 4(b), onde a cor é azulada, o coeficiente de correlação é muito baixo, baixando também a média geral das correlações para essa reanálise.

\section{Conclusões}

Para que um modelo regional represente de maneira satisfatória as condições de vento e, consequentemente, o potencial de geração eólica, é necesário dados de entrada que descrevam as carácterísticas geográficas e de clima da região. Uma alternativa é a utilização de conjuntos de dados de reanálises meteorológicas. Dessa forma o presente estudo visou verificar a validade das reanálises ERA-Interim e CFSR para descrever as características do campo de vento (magnitude e direção) para o estado do Rio Grande do Sul. Essa análise foi realisada através do cálculo do coeficiente de correlação dos dados de vento das reanálises com os dados das estações automáticas do INMET.

Os resultados demonstraram que os dados, tanto do ERA-Interim quanto do CFSR, representam de forma satisfatória os dados das estações do INMET para a magnitude do vento com um coeficiente de correlação médio maior que 0,6965 . Já para a direção do vento, os resultados foram insatisfatórios com coeficiente médio menor que 0,3642 . De maneira geral a reanálise com maior desempenho foi a CFSR, principalmente em relação a magnitude do vento como pode ser verificado na Figura 3. O ERA-Interim teve melhor resultado geral para a direção do vento. Porem o menor desempenho do CFSR se deve ao baixo coeficiente de correlação em três pontos específicos no mapa da Figura 4(b).

Verificando a sazonalidade das séries temporais analisadas, também observa-se que o período com melhores correlações encontra-se no inverno para ambas reanálises e que os piores períodos estão entre a primavera e o verão. Portanto, a conclusão geral obtida foi de que a utilização de reanálises para a previsão de campo de vento é viável, ao menos, como análise preliminar ou como condição de contorno para um modelo regional de previsão de vento, uma vez que a direção do vento não é bem representada pelas reanálises. Sendo que a melhor reanálise para a região de estudo, destre as duas analisadas, é a CFSR do NCEP. Desta forma, o próximo passo deste trabalho é utilizar os dados do CFSR para realizar um downscaling com o modelo WRF (Weather Research and Forecasting Model), passando então a verificar quais esquemas de camada limite apresentam melhor desempenho nesta tarefa.

\section{Agradecimentos}

Os autores agradecem ao Conselho Nacional de Desenvolvimento Científico e Tecnológico (CNPq), à Coor- 


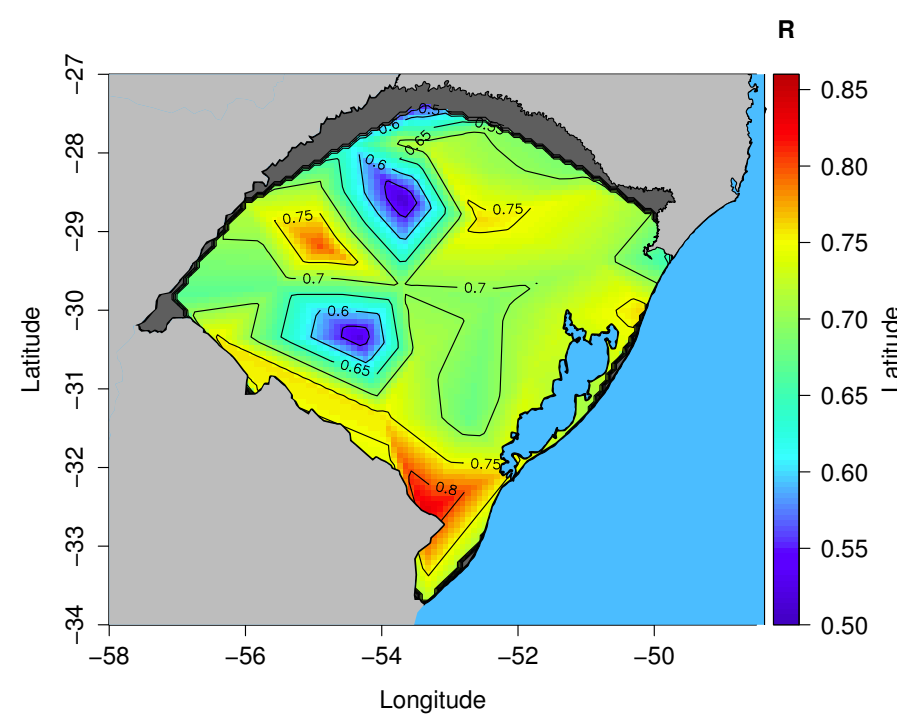

(a) ERA-Interim

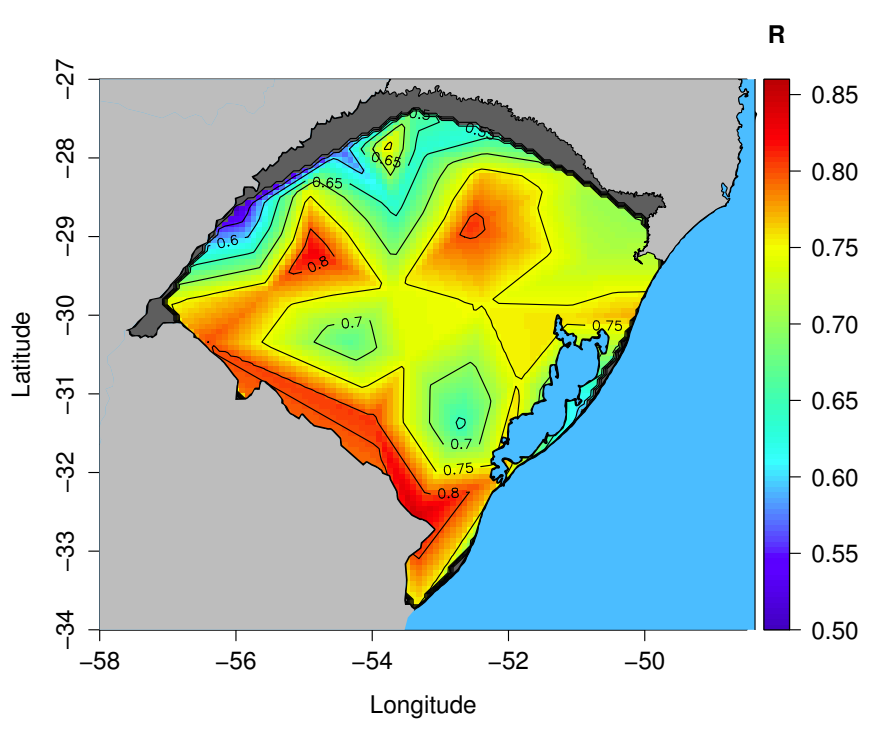

(b) CFSR

Figura 3: Coeficiente de correlação dos dados de magnitude do vento das estações automáticas do INMET com os dados das reanálises CFSR (Figura 3(b)) e ERA-Interim (Figura 3(a)) para o inverno.

denação de Aperfeiçoamento de Pessoal de Nível Superior (CAPES) e à Universidade Federal do Pampa (UNIPAMPA) pelo apoio financeiro. Os autores também agradecem ao Instituto Nacional de Meteorologia (INMET) pelo fornecimento de dados meteorológicos. O cluster computacional utilizado nas análises foi adquirido com recursos do edital Edital 02/2014 - PqG (Edital Pesquisador Gaúcho) da Fundação de Amparo a Pesquisa do Estado do Rio Grande do Sul (FAPERGS).

\section{Referências}

Carvalho, D., Rocha, A., Gómez-Gesteira, M., Santos, C. S. (2013). WRF wind simulation and wind energy production estimates forced by different reanalyses: Comparison with observed data for Portugal. Applied Energy, 117, 116-126.

CCEE (2015). Usinas eólicas mais que dobram a produçãoo de energia no primeiro semestre, URL http://www.ccee.org.br/portal/faces/ pages_publico/noticias-opiniao/noticias/ noticialeitura? contentid=CCEE_357460\& _afrLoop $=68521975034808 \# 40 \% 3 F$ contentid $\%$ 3DCCEE_357460\%26_afrLoop\%3D68521975034808\%26_ adf .ctrl-state\%3D17wx1e5can_45.

Dee, D., Uppala, S., Simmons, A., Berrisford, P., Poli, P., Kobayashi, S., Andrae, U., Balmaseda, M., Balsamo, G., Bauer, P., et al. (2011). The ERA-Interim reanalysis: Configuration and performance of the data assimila- tion system. Quarterly Journal of the Royal Meteorological Society, 137(656), 553-597.

Hanna, S. R. (1988). Hibrid Plume Dispersion Model (HPDM) Development and Evaluation. Journal of applied Meteorology, 28, 206-223.

INMET (2011). NOTA TÉCNICA No. 001/2011/SEGER/LAIME/CSC/INMET - Rede de Estações Meteorológicas Automáticas do INMET. MINISTÉRIO DA AGRICULTURA, PECUÁRIA E ABASTECIMENTO, URL http://www.inmet.gov.br/portal/css/ content/topo_iframe/pdf/Nota_Tecnica-Rede_ estacoes_INMET.pdf.

Kalnay, E., Kanamitsu, M., Kistler, R., Collins, W., Deaven, D., Gandin, L., Iredell, M., Saha, S., White, G., Woollen, J., Zhu, Y., Leetmaa, A., Reynolds, R., Chelliah, M., Ebisuzaki, W., Higgins, W., Janowiak, J., Mo, K. C., Ropelewski, C., Wang, J., Jenne, R., Joseph, D. (1996). The ncep/ncar 40-year reanalysis project. Bulletin of the American Meteorological Society, 77(3), 437-471.

Liléo, S., Petrik, O. (2011). Investigation on the use of NCEP/NCAR, MERRA and NCEP/CFSR reanalysis data in wind resource analysis. European Wind Energy Association.

Pinto, M. d. O. (2013). Fundamentos de Energia Eólica. LTC, Rio de Janeiro, Brasil.

Rienecker, M. M., Suarez, M. J., Gelaro, R., Todling, R., Bacmeister, J., EmilyLiu, Bosilovich, M. G., Schubert, S. D., Takacs, L., Kim, G. K., Bloom, S., Chen, 
$\mathbf{R}$

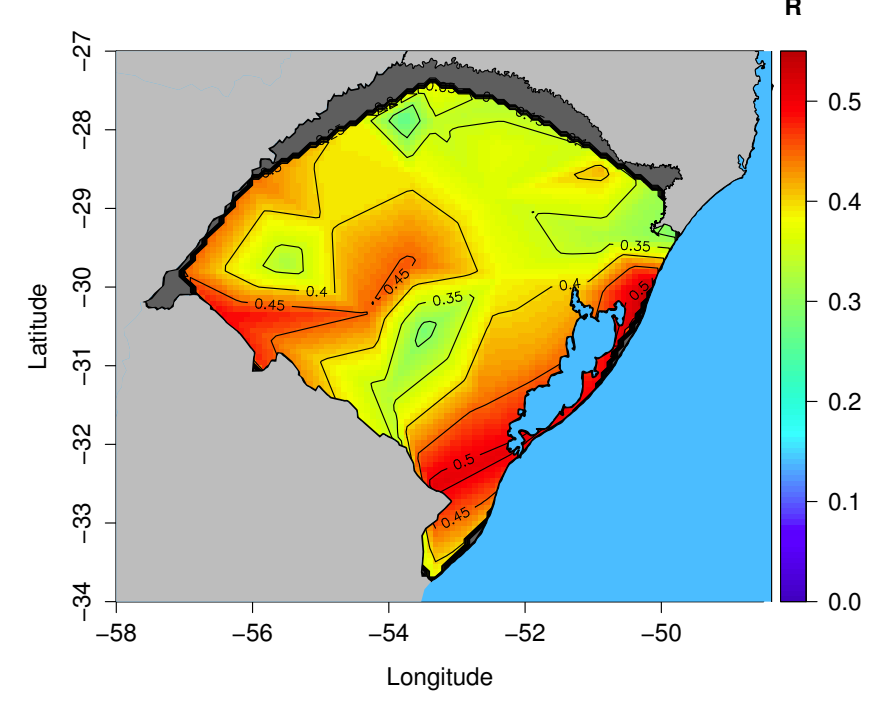

(a) ERA-Interim

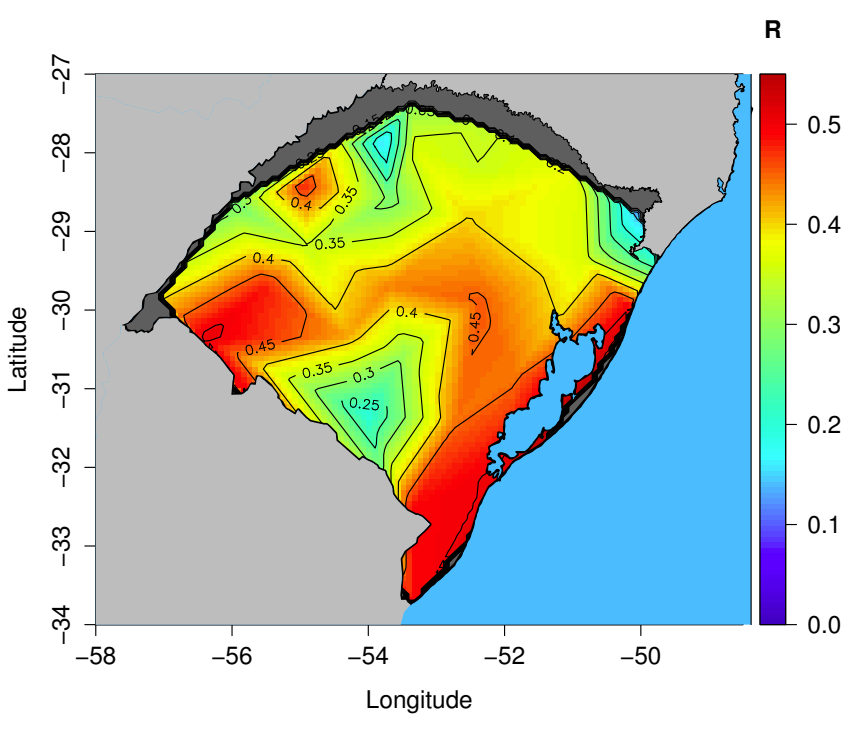

(b) CFSR

Figura 4: Coeficiente de correlação dos dados da direção do vento das estações automáticas do INMET com os dados das reanálises CFSR (Figura 4(b)) e ERA-Interim (Figura 4(a)) para o inverno.

J., Collins, D., Conaty, A., da Silva, A., Gu, W., Joiner, J., D.Koster, R., Lucchesi, R., Molod, A., Owens, T., Pawson, S., Pegion, P., Redder, C. R., Reichle, R., Robertson, F. R., Ruddick, A. G., Sienkiewicz, M., Woollen, J. (2011). MERRA: NASA's Modern-Era Retrospective Analysis for Research and Applications. Journal of Climate, 24, 3624-3648.

Saha, S., Moorthi, S., Pan, H. L., Wu, X., Wang, J., Nadiga, S., ck Tripp, P., Kistler, R., en, J. W., d Behringer, D., Liu, H., Stokes, D., Grumbine, R., Gayno, G., Wang, J., Hou, Y. T., Chuang, H. y., Juang, H. M. H., Sela, J., Iredell, M., Treadon, R., Kleist, D., t, P. V. D., Keyser, D., Derber, J., Ek, M., e Meng, J., Wei, H., an Yang, R., Lord, S., van den Dool, H., Kumar, A., u Wang, W., Long, C., iah, M. C., Xue, Y., n Huang, B., Schemm, J. K., ey Ebisuzaki, W., Lin, R., Xie, P., Chen, M., Zhou, S., Higgins, W., Zou, C. Z., Liu, Q., Chen, Y., Han, Y., Cucurull, L., Reynolds, R. W., Rutledge, G., Goldberg, M. (2010). The NCEP climate forecast system reanalysis. American Meteorological Society, 91, 1015-1057.

Stensrud, D. J. (2007). Parameterization schemes: keys to understanding numerical weather prediction models. Cambridge University Press. 